\title{
Intramuscular Myxoma of the Paraspinal Musculature
}

\author{
Simon Stinchcombe ${ }^{\mathrm{a}, \mathrm{b}}$, Rohit Kochhar ${ }^{\mathrm{a}}$, Dilip Malkan ${ }^{\mathrm{a}}$
}

\begin{abstract}
Intramuscular myxomas are uncommon, benign tumors of mesenchymal origin most noted for their occurrence in the left atrium. We present a rare case of intramuscular myxoma involving the paraspinal muscles of an 80-year-old woman, presenting with longstanding lower back pain. There was no focal neurological deficit. Imaging revealed a large lobulated paraspinal mass, with heterogeneous rim enhancement, causing adjacent bony changes and intraspinal extension. Open spinal biopsy with debridement and drainage of a suspected paraspinal abscess was done. The diagnosis of an intramuscular myxoma was however confirmed on histopathology. Intramuscular myxomas, particularly those occurring in distinctly uncommon locations, with aggressive features, can pose diagnostic challenges and should be included in the differential diagnosis of all paraspinal cystic lesions.
\end{abstract}

Keywords: Myxoma; Paraspinal musculature; MR imaging

\section{Introduction}

Intramuscular myxomas are rare soft tissue myxoid tumors of mesenchymal origin. They are characterized clinically by slow growth with minimal symptoms and histologically by an abundant myxoid matrix and a paucity of spindle-shaped stromal cells. Stout [1] in 1948 first established intramuscular myxoma as a distinct entity, and in his original study, only $3 \%$ of the lesions were intramuscular in origin.

Manuscript accepted for publication August 16, 2010

${ }^{\text {a}}$ Kings Mill Hospital, UK

${ }^{\mathrm{b}}$ Corresponding author: Kings Mill Hospital, UK.

Email: Simon.Stinchcombe@sfh-tr.nhs.uk

doi:10.4021/jmc37w
Characteristic imaging features of this benign neoplasm have been described, which are of importance in suggesting a preoperative diagnosis, as intramuscular myxomas can mimic other benign as well as malignant myxoid lesions [2$4]$.

We present one such diagnosed case of an intramuscular myxoma involving the paravertebral muscles, a distinctly uncommon location with certain atypical imaging features, including changes in adjacent bone, which gave the appearance of a more aggressive process.

\section{Case Report}

An 80-year-old woman presented with a painful lower back for four months, with increasing severity of pain over the last two to three weeks. She had a history of weight loss of three and a half stones over the last six months. On examination, paraspinal tenderness was elicited. However, no definite mass was felt. There was no focal neurology. Significant past history included pulmonary tuberculosis (TB), right above knee amputation for osteosarcoma twenty six years ago and a left total knee replacement ten years ago. There was no previous history of spinal surgery and she had received no intramuscular injections. Her blood results revealed hemoglobin of $13.1 \mathrm{gm} \%$ and a white cell count of 7.1.

The chest X-ray was grossly unremarkable, but for a Gohns focus, with no evidence of metastases. X-ray of the lumbo-sacral spine revealed no bony destruction. MR examination showed a large multiloculated, predominantly cystic mass, involving the paravertebral muscles bilaterally from the L2 to S1 vertebral levels (Fig. 1a, b). There was involvement of the right facet joint at the L5 level, with destruction of the right lamina and extension into the central spinal canal (Fig. 1c, d, e). Following contrast administration, there was a moderately intense peripheral rim enhancement as well as a patchy enhancement of the intraspinal component (Fig. 1f, g). The imaging features of peripheral enhancement and laminar destruction were thought to be due to a moderately aggressive process and in keeping with the presentation and clinical history, including a past history of pulmonary TB, a periarticular spinal abscess, possibly secondary to TB was 

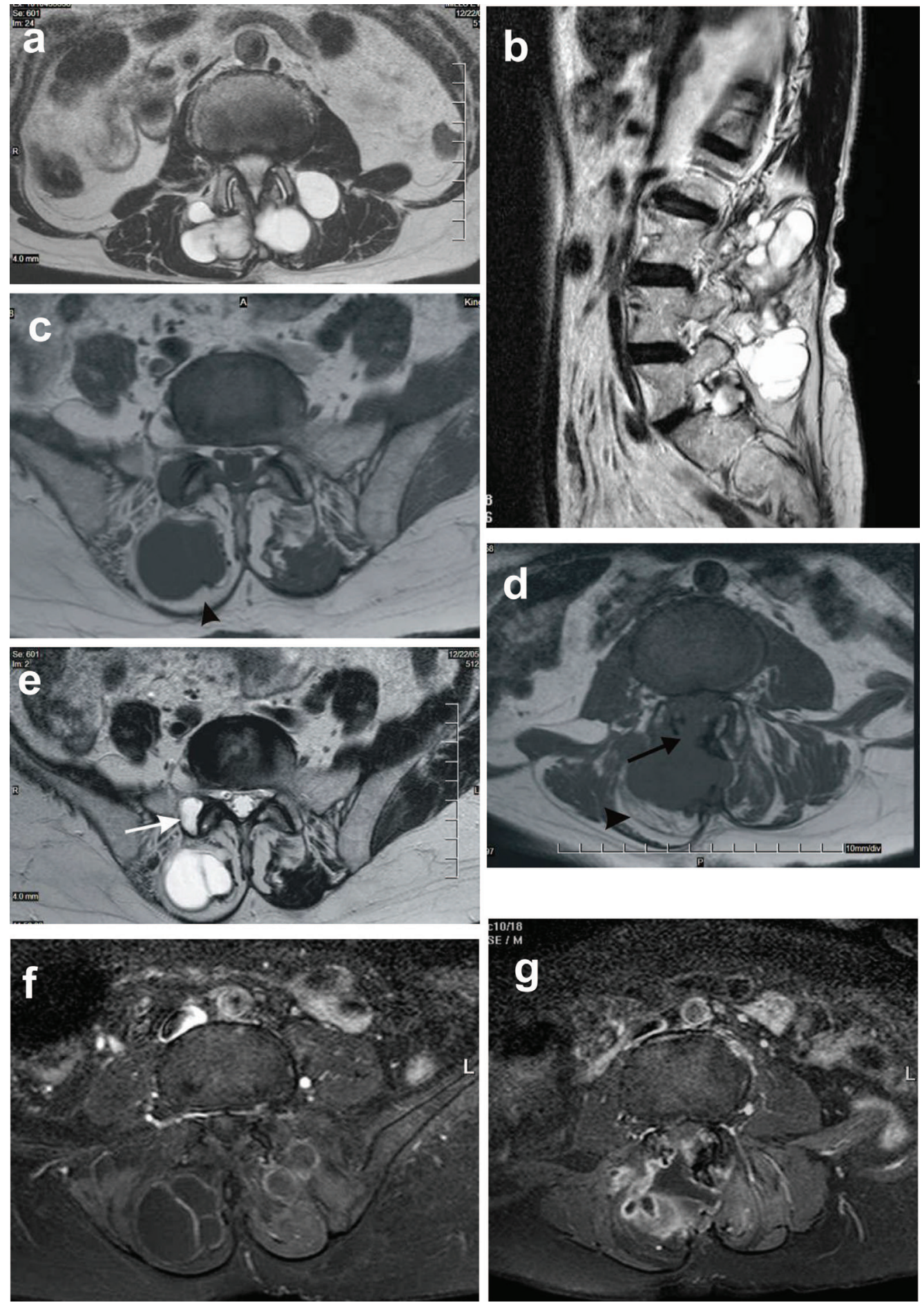

Figure 1. $\mathbf{a}$ and b: Axial and sagittal T2 weighted images showing a large, markedly T2 hyperintense multiloculated mass lesion involving the paraspinal muscles bilaterally (1a) and extending from L2 to S1 vertebral levels (1b); c and d: Axial T1 weighted images showing increased signal in the adjacent paraspinal muscles (arrow heads), in keeping with fatty atrophy. There is destruction of the right lamina with extension into the central spinal canal (arrow in d); e: Note is made of involvement of the right facet joint with cystic change as seen by the increased signal on these T2 weighted images (arrow); f and g: Post contrast axial T1 weighted images showing peripheral rim and septal enhancement. In addition there is patchy heterogeneous enhancement of the intraspinal component. 


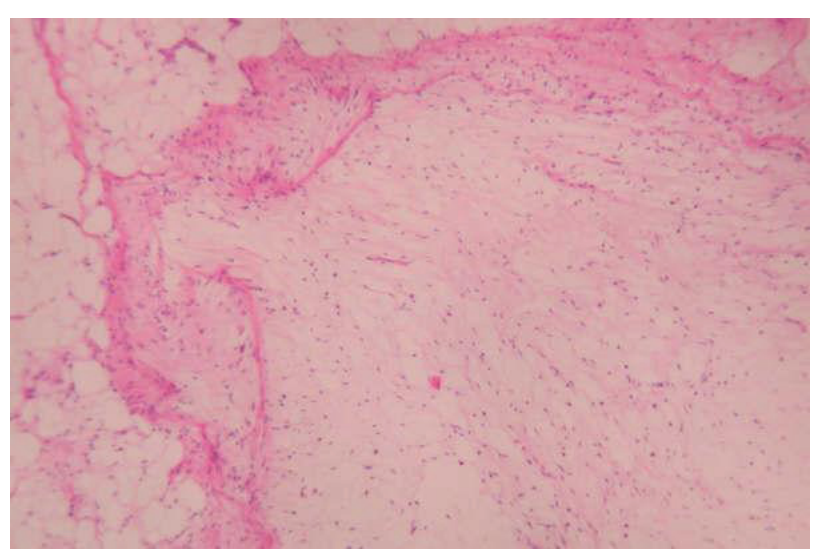

Figure 2. Low power photomicrograph of intramuscular myxoma in an 80-year-old woman showing hypocellular myxoma with small stellate cells. Note the lesion is pushing into local adipose tissue (seen left) (Original magnification X 50).

considered as the most likely diagnosis.

Subsequently an open spinal biopsy with debridement and drainage of the suspected paraspinal abscess was done, using a dorsal incision over L2 to L5 levels. A large gelatinous mass was seen in the posterior paraspinal muscles which contained loculated material that was neither fluid nor pus like in appearance. After debulking the site was packed, a drain was put, and samples were sent for microbiology and histopathology.

The culture of the tissue sample sent for microbiology was sterile and in particular, there was no evidence of Mycobacterium Tuberculosis. Macroscopic examination revealed multiple fragments of white myxoid tissue, some of which were attached to bone. A multiloculated cyst measuring $4.0 \mathrm{x}$ $4.0 \times 3.0 \mathrm{~cm}$ containing mucoid material and a fibrocartilagenous base of 4.0 × $3.0 \mathrm{~cm}$ was seen. Microscopic examination confirmed a multiloculated, highly myxoid, hypocellular lobulated lesion, with focal cyst formation. Myxoid material was present within the cyst wall, which was composed of fibrous connective tissue. The cells were small, spindly or stellate, with no significant pleomorphism or mitotic rate. There was no necrosis. The findings were consistent with a benign intramuscular myxoma (Fig. 2).

\section{Discussion}

Intramuscular myxomas are relatively rare soft tissue tumors. In a descending order of frequency, the large muscles of the thigh, shoulder, buttocks or upper arm are most commonly affected [5]. A small number of cases occur in intermuscular, subcutaneous or juxtaarticular locations. Intramuscular myxomas occurring in paraspinal musculature are distinctly uncommon with only a few isolated case reports [6]. Women are more frequently affected than men, with a ratio of 1.4:1, with a mean patient age of 52 years [7].

A typical intramuscular myxoma appears as a well-defined ovoid lesion showing fluid signal intensity, correlating with the high water content of mucin seen histologically [7]. Typically a peritumoral fatty rim is visible on T1-weighted MR and increased signal in keeping with edema, is seen in the adjacent muscle on T2-weighted or fluid sensitive MR sequences. It has been suggested that the latter two imaging features are the most reliable in differentiating intramuscular myxomas from other myxoid soft tissue lesions [3]. Intramuscular myxomas tend to be slow growing, usually lack a complete pseudocapsule and infiltrate the adjacent atrophic and edematous muscle. The fatty rind between the tumor and the adjacent muscle interface is due to reactive fat as a result of muscle atrophy [8] and may be seen in up to $71 \%$ of cases [7]. Myxomas are typically composed of a hypo to avascular myxoid stroma but show variable enhancement patterns, which are usually heterogeneous and hyperintense [4]. Intramuscular myxomas are sonographically hypoechoic rather than anechoic, reflecting the true solid architecture of these lesions and show low attenuation on CT [7].

Histologically, intramuscular myxomas are composed of a few spindle-shaped cells interspersed within an abundant amount of mucoid substance, overlying a loose reticulin framework. They are characterized by a paucity of cells, diminished vascularity and minimal mitotic figures [9].

Differential diagnosis of intramuscular myxomas includes other benign lesions which have a high water content namely synovial cysts, bursa, and ganglia. However, the majority of synovial cysts, bursa and ganglia are true cystic lesions, are anechoic on ultrasound, occur in typical locations and are intermuscular, as opposed to the intramuscular myxomas.

An intramuscular cystic mass with irregular enhancement raises the possibility of infection although the clinical history is often helpful in distinguishing an abscess from other cystic lesions. Chronic infections like tubercular spondylitis and lumbar facet joint infections leading to septic arthritis are known to be associated with paraspinal abscesses $[10,11]$. Tubercular spondylitis is often associated with bony deformity of the spine and classic findings of spodylodiscitis [12]. Imaging findings of facet joint infection include effusion into a widened facet joint and there may be previous history of facet joint injection.

In addition the differential diagnosis also includes neurogenic neoplasms, myxoid liposarcomas and myxoid malignant fibrous histiocytoma. Neurogenic tumors are also intermuscular, often showing an entering and exiting nerve on imaging and a target sign on T2-weighted MR imaging [13]. Myxoid liposarcomas, especially if located intramuscularly, tend to mimic the imaging, surgical and pathological appearance of intramuscular myxomas [14]. Differentiating the two 
is of great clinical importance as myxomas have a benign course with no tendency for recurrence or metastases [8]. Myxoid liposarcomas are usually intermuscular, and often contain a small amount of intrinsic fat on MR imaging [15]. Myxoid malignant fibrous histiocytoma, like myxomas, are intramuscular lesions, but have a more heterogeneous appearance, with areas of hemorrhage and nodular regions showing strong contrast enhancement [16].

Treatment is usually with at least marginal excision of the mass. However, as it may be difficult to preoperatively differentiate intramuscular myxoma from malignant myxoid tumors, excision with wide margins is often favored [17].

Our case demonstrated certain atypical features that made it difficult to make the correct diagnosis preoperatively. It is extremely uncommon for myxomas, which have a predilection for the extremities, to involve the paraspinal muscles. Bony destruction and intraspinal extension of myxomas occurring in a paravertebral location have only rarely been reported before [6].

Soft tissue myxomas have been known to be associated with fibrous dysplasia in the same anatomical region in a rare disease known as Mazabraud's syndrome [18]. The associated bony lesions in our case in the form of cystic change and destruction of the lamina and extension into the spinal canal were highly atypical for fibrous dysplasia and there was no evidence of the same on histopathology.

Imaging features suggested chronic infection with septic arthritis and periarticular abscess formation as the most likely cause. The patchy enhancement of the intraspinal component and peripheral rim enhancement further supported this diagnosis. The combination of a periarticular cystic mass with associated periarticular intraosseous cystic change also raised the possibility of a ganglion cyst, although this diagnosis was considered unlikely. In retrospect however, the presence of a peritumoral fat rim could have alerted us to the correct diagnosis.

Intramuscular myxomas, particularly those occurring in atypical locations, pose diagnostic challenges. They should be included in the differential diagnosis of all intramuscular cystic lesions even in uncommon locations, and even if coexistent intraosseous cystic or apparently destructive lesions are present. In all intramuscular cystic lesions the images should be carefully examined for a peritumoral fat rim which could indicate the presence of a myxoma.

\section{References}

1. Stout AP. Myxoma, the tumor of primitive mesenchyme. Ann Surg 1948;127(4):706-719.

2. Schwartz HS, Walker R. Recognizable magnetic resonance imaging characteristics of intramuscular myxoma. Orthopedics 1997;20(5):431-435.

3. Bancroft LW, Kransdorf MJ, Menke DM, O’Connor
MI, Foster WC. Intramuscular myxoma: characteristic MR imaging features. AJR Am J Roentgenol 2002;178(5):1255-1259.

4. Luna A, Martinez S, Bossen E. Magnetic resonance imaging of intramuscular myxoma with histological comparison and a review of the literature. Skeletal Radiol 2005;34(1):19-28.

5. Ly JQ, Bau JL, Beall DP. Forearm intramuscular myxoma. AJR Am J Roentgenol 2003;181(4):960.

6. Guppy KH, Wagner F, Tawk R, Gallagher L. Intramuscular myxoma causing lumbar radiculopathy. Case report and review of the literature. J Neurosurg 2001;95(2 Suppl):260-263.

7. Murphey MD, McRae GA, Fanburg-Smith JC, Temple HT, Levine AM, Aboulafia AJ. Imaging of soft-tissue myxoma with emphasis on CT and MR and comparison of radiologic and pathologic findings. Radiology 2002;225(1):215-224.

8. Kindblom LG, Stener B, Angervall L. Intramuscular myxoma. Cancer 1974;34(5):1737-1744.

9. Iwasko N, Steinbach LS, Disler D, Pathria M, Hottya GA, Kattapuram S, Varma DG, et al. Imaging findings in Mazabraud's syndrome: seven new cases. Skeletal Radiol 2002;31(2):81-87.

10. Okada F, Takayama H, Doita M, Harada T, Yoshiya S, Kurosaka M. Lumbar facet joint infection associated with epidural and paraspinal abscess: a case report with review of the literature. J Spinal Disord Tech 2005;18(5):458-461.

11. $\mathrm{Ng} \mathrm{AW}, \mathrm{Chu} \mathrm{WC}, \mathrm{Ng} \mathrm{BK}, \mathrm{Li}$ AM. Extensive paraspinal abscess complicating tuberculous spondylitis in an adolescent with Pott kyphosis. Clin Imaging 2005;29(5):359-361.

12. De Backer AI, Mortele KJ, Vanschoubroeck IJ, Deeren D, Vanhoenacker FM, De Keulenaer BL, Bomans P, et al. Tuberculosis of the spine: CT and MR imaging features. JBR-BTR 2005;88(2):92-97.

13. Murphey MD, Smith WS, Smith SE, Kransdorf MJ, Temple HT. From the archives of the AFIP. Imaging of musculoskeletal neurogenic tumors: radiologic-pathologic correlation. Radiographics 1999;19(5):1253-1280.

14. Weiss SW, Goldblum JR. Enzinger and Weiss's soft tissue tumors, 4th ed. St. Louis: Mosby, 2001: 1419 -1481.

15. Sung MS, Kang HS, Suh JS, Lee JH, Park JM, Kim JY, Lee HG. Myxoid liposarcoma: appearance at MR imaging with histologic correlation. Radiographics 2000;20(4):1007-1019.

16. Murphey MD, Gross TM, Rosenthal HG. From the archives of the AFIP. Musculoskeletal malignant fibrous histiocytoma: radiologic-pathologic correlation. Radiographics 1994;14(4):807-826; quiz 827-808.

17. Charron P, Smith J. Intramuscular myxomas: a clinicopathologic study with emphasis on surgical management. Am Surg 2004;70(12):1073-1077. 
18. Kabukcuoglu F, Kabukcuoglu Y, Yilmaz B, Erdem Y, Evren I. Mazabraud's syndrome: intramuscular myxo- ma associated with fibrous dysplasia. Pathol Oncol Res 2004;10(2):121-123. 